\title{
Characterization of Zircaloy-4 Corrosion Films using Microbeam Synchrotron Radiation
}

\author{
David J. Spengler ${ }^{1,4^{*}}$, Arthur T. Motta ${ }^{1}$, Ram Bajaj ${ }^{2}$, John Seidensticker ${ }^{2}$, \\ Zhonghou Cai ${ }^{3}$
}

${ }^{1}$ Department of Mechanical and Nuclear Engineering, The Pennsylvania State University, University Park, PA, 16802.

${ }_{2}^{2}$ Bettis Atomic Power Laboratory, 814 Pittsburgh McKeesport Blvd., West Mifflin, PA 15122.

${ }^{3}$ Advanced Photon Source, Argonne National Laboratory, Argonne, IL, 60439

${ }^{4}$ Currently at Knolls Atomic Power Laboratory, 2401 River Road, Niskayuna, NY 12309

*Corresponding Author- Email Address: dspen5106@gmail.com Phone: +15186125105

Address: 2401 River Road: Mail Stop 114, Niskayuna, NY 12309

Abstract: A study of the oxide layers formed in $360^{\circ} \mathrm{C}$ and $316{ }^{\circ} \mathrm{C}$ water on Zircaloy-4 samples has been performed in an attempt to help answer fundamental questions about oxide protectiveness, growth mechanisms, and the nature of oxide growth during autoclave corrosion. Two different oxide thicknesses -12 and 39.5 microns - were investigated. Microbeam synchrotron radiation diffraction and fluorescence techniques with an x-ray beam size of 0.2 microns were used to characterize oxide in cross sections to determine the oxide phase content, grain size, texture, and orientation relationships as a function of through-thickness from the oxide-metal interface.

The results confirm that the oxide is comprised primarily of monoclinic $\mathrm{ZrO}_{2}$, with tetragonal $\mathrm{ZrO}_{2}$ present in small amounts. The observed diffraction peaks are consistent with monoclinic phases having a strong fiber texture with the $200_{m}$ plane aligned with the oxide-metal interface, and with the $011 \mathrm{~m}$ plane closely aligned with the transversenormal $(\mathrm{T})$ plane.

The fraction of bulk tetragonal phase increased in the region located within one transition thickness near the oxide-metal interface. A strong periodicity was seen in oxide intensity from both the monoclinic and tetragonal phases corresponding to an oxide transition thickness of 1.8 to 1.9 microns. The grain size of the tetragonal phase was determined to be smaller than the monoclinic phase, and the grain size for the monoclinic phase decreased starting at a distance of approximately one transition layer from the oxide-metal interface. The relative amounts of monoclinic peak broadening due to strain and grain size were calculated, the former being approximately constant, while the latter decreased with increasing distance from the oxide-metal interface, corresponding to an increase in grain size. These findings are compared to previously performed microbeam diffraction experiments on Zircaloy-4 and other Zr-based alloys. 


\section{Introduction}

Waterside corrosion of zirconium alloys in pressurized water reactor (PWR) primary system water has the potential to become a life-limiting degradation mechanism as the nuclear industry subjects fuel rods to increasingly higher burnup in an effort to increase cycle length and improve capacity factor. Modern zirconium alloys such as ZIRLO ${ }^{\mathrm{TM}}$ and $\mathrm{M} 5^{\mathrm{TM}}$ show improved corrosion performance compared to previous generation alloys such as Zircaloy-4; however, the underlying reasons for this improved performance are poorly understood. To better predict long-term corrosion behavior, it is important that the mechanisms for the degradation of the fuel cladding are fully understood as the materials are progressively pushed to more severe duty conditions.

The oxide layer formed on zirconium alloys during waterside corrosion is understood to have a protective nature that varies periodically during corrosion as a result of cyclic transitions in the oxidation kinetics. While the influence of the transition on the formation of new oxide is not well understood, it is clear that a brief loss of protectiveness in the oxide layer occurs at transition.

Many studies have been undertaken in an attempt to explain the oxide transition mechanism, the results of which can be summarized into two main explanations. The "mechanical failure" hypothesis explains the transition as the result of lateral cracking at the oxide-metal interface, which originates from the stresses generated during oxide growth and allows diffusing species easy access to the metal substrate [1-5]. Lateral cracks in the oxide are frequently observed in scanning and transmission electron microscopy. However, it is not clear how lateral cracks at the interface can accelerate oxidation without paths from the environment, through the cracks, to the oxide-metal interface. A second explanation, called the "formation of interconnected porosity" hypothesis, postulates that the buildup of stresses leads to the formation of micro cracks and micro pores throughout the oxide [6-11]. When these micro pores and cracks connect, they provide a pathway for the diffusing species to easily migrate through the oxide to the oxide-metal interface. It is still unknown how the micro pores and micro cracks are generated and how they connect in a concerted manner to cause the clear transition in corrosion kinetics apparent in weight-gain versus time corrosion curves.

Previous studies [12-15] have provided an experimental basis for a third mechanism that attempts to bridge the gap between the mechanical failure and interconnected porosity mechanisms. The mechanism is described in detail in [13] and is summarized in the following paragraph.

At the start of oxidation, small oxide grains nucleate at the metal surface. This initial layer is comprised of both monoclinic and tetragonal phases, with a relatively large proportion of tetragonal grains that have been stabilized by a combination of high stresses and oxide sub-stoichiometry. A fraction of the newly formed tetragonal grains will be properly oriented for growth, and as such will self-select to grow. As the tetragonal oxide grains grow they become columnar, and after reaching a critical 
column length will transform into the monoclinic phase while maintaining the same orientation. Improperly oriented tetragonal or monoclinic grains will remain as small grains embedded in the oxide. The columnar monoclinic grains will proceed to grow into the metal. During the growth process, small mismatches in the orientation of the column with the preferred orientation and surrounding grains causes stress to accumulate locally. As they grow the columns become progressively more misaligned, and, at an aspect ratio of roughly 4 to 5 , the stress has built up to a level that grain renucleation is energetically favorable for continued grain growth. At this point renucleation of a new grain along the leading growth edge occurs. When re-nucleation occurs, the newly created grains are small, equiaxed, and have a higher percentage of the tetragonal phase. The re-nucleated grains can then grow and form a new column of monoclinic oxide if properly oriented, or remain embedded in the oxide. On the scale of the entire oxide thickness, a buildup of bulk stresses will eventually cause the oxide to crack and undergo a global transition (as measured on a global scale using the weight gain). The cracking occurs laterally, as observed by many authors, and as expected from the development of in-plane stresses during oxide growth. Such lateral cracks may then cause transition by causing the interconnection of pre-existing porosity and allowing water to access the interface. This global transition causes a large scale renucleation of oxide grains through a similar mechanism as described above for the initial corrosion cycle. At this point the entire process restarts, and the first step is repeated.

In this work, the structure of the oxide as a function of distance from the oxide-metal interface was studied using micro-beam synchrotron radiation. These studies were performed on oxides grown on Zircaloy-4 in pure water. The objective of this study is to compare and relate the structure of the oxide to currently proposed transition mechanisms in order to further the fundamental understanding of the oxide transition mechanism and its influencing factors.

\section{Experimental Methods}

\subsection{Alloys and Samples Examined}

Coupons of Zircaloy- 4 were corroded in water at $360{ }^{\circ} \mathrm{C}$ and $316{ }^{\circ} \mathrm{C}$ in autoclaves. The coupons were cut from heats of alpha-annealed cold-worked and stress-relieved plates, and had alloying element and impurity compositions meeting ASTM B-350 requirements $[16,17]$. Typical specimen dimensions were $25 \mathrm{~mm} \times 25 \mathrm{~mm} \times 1 \mathrm{~mm}$ and prior to corrosion were pickled using a solution of 39 vol. $\% \mathrm{HNO}_{3}$ plus $3.5 \mathrm{vol} . \% \mathrm{HF}$ with the remainder water [17].

Two Zircaloy-4 specimens designated L1581 and N2511 were corroded at $680^{\circ} \mathrm{F}(360$ ${ }^{\circ} \mathrm{C}$ ) for 1260 days and $600{ }^{\circ} \mathrm{F}\left(316^{\circ} \mathrm{C}\right)$ for 3113 days, respectively, as shown in Table 1. The total time at temperature for L1581 was 1260 days which resulted in a final measured weight gain of $570 \mathrm{mg} / \mathrm{dm}^{2}$ and a corresponding average oxide thickness of 
$39.5 \mu \mathrm{m}$ [16]. The final measured weight gain of sample $\mathrm{N} 2511$ was $177 \mathrm{mg} / \mathrm{dm}^{2}$ which corresponded to an average oxide thickness of $12.0 \mu \mathrm{m}$. Corrosion kinetics curves for L1581 and N2511 are shown in Figure 1. Oxide transitions can be identified by a sharp change in the slope of the weight gain curve. A total of three kinetic transitions are visible in the L1581 weight gain curve, followed by a linear corrosion regime with no discernible periodic transitions. Sample N2511 shows five transitions in its weight gain curve, with a possible sixth (at $\sim 3100$ days) visible as well.

Table 1 - Summary of sample characteristics and corrosion data for the two coupons highlighted in this study.

\begin{tabular}{cccccc}
\hline $\begin{array}{c}\text { Coupon } \\
\text { Designation }\end{array}$ & $\begin{array}{c}\text { Oxide Thickness } \\
(\boldsymbol{\mu m})\end{array}$ & $\begin{array}{c}\text { Corrosion } \\
\text { Time }\end{array}$ & $\begin{array}{c}\text { Corrosion } \\
\text { Temp. }\left({ }^{\circ} \mathbf{C}\right)\end{array}$ & $\begin{array}{c}\text { Material } \\
\text { Type }\end{array}$ & $\boldsymbol{f}_{\boldsymbol{N}}$ \\
\hline $\mathrm{N} 2511$ & 12.0 & 3113 days & 316 & Zircaloy-4 & 0.6 \\
L1581 & 39.5 & 1260 days & 360 & Zircaloy-4 & 0.6 \\
\hline
\end{tabular}

$f_{N}$ refers to the normal Kearns Factor, which is the resolved fraction of basal poles aligned in the normal direction

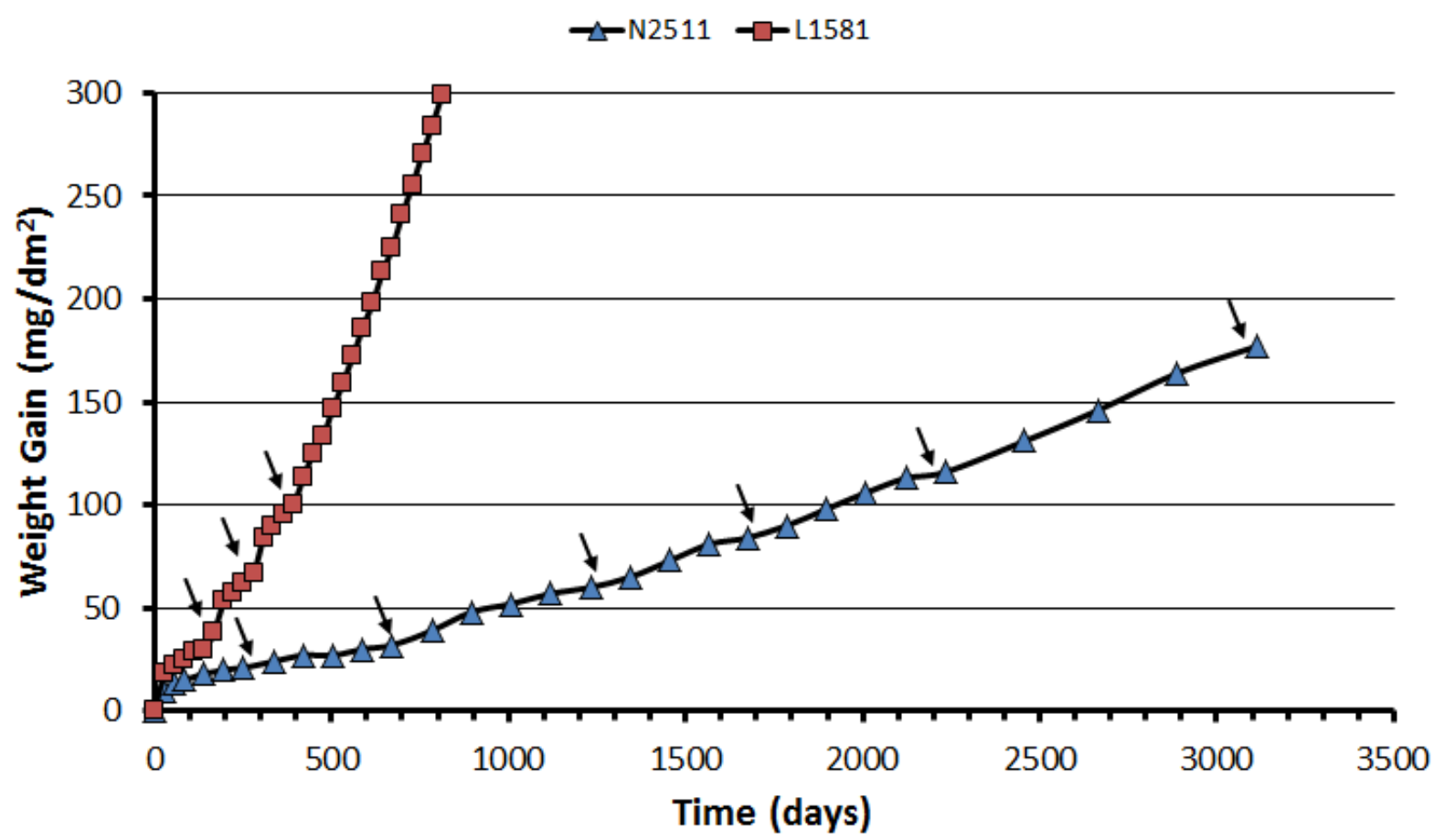

Figure 1: Weight gains for Zircaloy-4 specimens during autoclave corrosion in water. Kinetic transitions are labeled with black arrows. Note that the $y$-axis has not been extended to the maximum weight gain for $\mathrm{L} 1518$ of $570 \mathrm{mg} / \mathrm{dm}^{2}$ to make the early kinetic transitions more visible.

The features of the oxide layers were briefly examined in cross-section using a scanning electron microscopy (SEM) in secondary electron mode. The preparation techniques will be described in a later section. The oxide and its features are clearly 
visible in the SEM, with the primary contrast mechanism being the difference in average atomic weight between $\mathrm{ZrO}_{2}$ and zirconium. Figure 2 presents secondary electron SEM images of the oxide layers from L1581 and N2511 taken using secondary electrons at an accelerating voltage of $5 \mathrm{kV}$.

The oxide layers that grow on Zircaloy-4 exhibit several distinct characteristics observable in the SEM that can be compared between samples of different oxide thickness. Periodic cracking is visible in both oxide layers. Each layer of cracking roughly corresponds to one oxide transition layer, with the number of crack layers roughly equal to the number of oxide transitions, although the transitions may not be readily visible through weight gain data due to the periodic cycles of cracking becoming out of phase with one another past the first several cycles. The oxide-metal interface is wavy, with periodic regions of thicker and thinner oxide. This characteristic is seen in nearly all autoclave-grown oxides on zirconium alloys and is an indication that the oxide growth into the metal is uneven in the lateral direction. Veins of unbroken oxide are visible in the thick oxide grown on L1581. The veins are paths of unbroken oxide propagating vertically through the oxide thickness that contain no visible cracks. The veins are observed to take a tortuous route through the oxide thickness from the surface, and terminate at the points of minimum thickness in the wavelike shape of the oxide-metal interface.

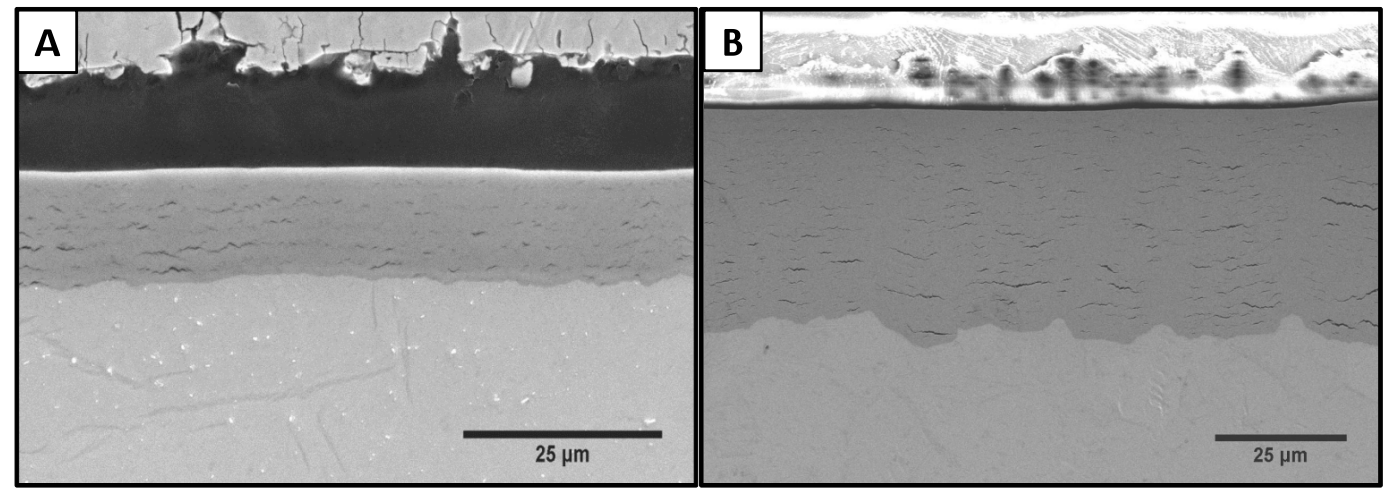

Figure 2 - Secondary electron SEM image of the oxide layers for sample (A) N2511, (B) L1581.

\subsection{Synchrotron Sample Preparation}

Oxidized samples were mounted in cross-section and polished to a mirror finish for examination with synchrotron radiation. The sample preparation techniques used were similar to those described in $[12-15,18]$ and are briefly summarized here. First, corrosion coupons were cut using a diamond saw to obtain small rectangular strips roughly $2 \mathrm{~mm}$ wide and $10 \mathrm{~mm}$ long. The strips were then mechanically thinned, which sacrifices the oxide layer on one side, to facilitate insertion into a slotted molybdenum rod, which was subsequently inserted into a $3 \mathrm{~mm}$ diameter brass tube. The entire assembly was secured together using Epo-Tek $® 353 \mathrm{ND}$ epoxy. The molybdenum rod helps to maintain the mechanical stability of the sample during polishing and to reduce 
the volume fraction of epoxy used. After the epoxy was heat-cured using a hot plate, the assembled rods were sliced into discs roughly $3 \mathrm{~mm}$ thick using a low speed diamond saw.

The cross-sectional discs were then polished using progressively finer grit silicon carbide paper and adhesive polishing discs, beginning with 180 grit and finishing at 1200 grit. A final polish was accomplished using 1- $\mu \mathrm{m}$ diamond paste followed by a $0.05-\mu \mathrm{m}$ colloidal silica solution.

\subsection{Synchrotron Radiation Diffraction Experiments}

Beamline 2-ID-D at the Advanced Photon Source (APS) at Argonne National Laboratory (ANL) was used to conduct a high resolution sub-micron $x$-ray study of these oxide layers. The $x$-rays are initially produced by the deceleration of synchrotron-accelerated electrons using an undulator. Once emitted from the undulator, the x-rays travel through various plates that define the beam energy and focus. This allows the x-ray energy (or wavelength) to be varied between 5 and $32 \mathrm{keV}$, and can result in a final focused beam spot size of $0.2 \mu \mathrm{m} \times 0.2 \mu \mathrm{m}$. The high brilliance/intensity of the beam at the APS ensures that there is sufficient signal reaching the detectors even after focusing the beam down to this size.

In addition to its high spatial resolution, another major advantage of beamline 2-ID-D is the ability to simultaneously capture elemental and crystallographic data through x-ray fluorescence and diffraction. Fluorescence data is collected by defining energy regions of interest (ROI) corresponding to the $\mathrm{K}$ or $\mathrm{L}$ lines of a specific element of interest, then recording counts from each $\mathrm{ROI}$ using a solid state detector when the sample fluoresces under the incident synchrotron x-rays. These were defined for $\mathrm{Zr}$ and $\mathrm{Sn} \mathrm{L}$ lines, as well as $\mathrm{Fe}, \mathrm{Cr}$, and $\mathrm{Ni} \mathrm{K}_{\alpha}$ lines. Simultaneously, diffraction data are collected using a 2-D CCD detector positioned at a fixed two-theta angle from the incident beam, which allows the peaks of interest to be recorded in the CCD camera.

The experiment was performed by raster scanning the beam in steps through the thickness of the oxide layer (referred to as a 'scan'). This process allowed elemental and microstructural information to be obtained as a function of position in the oxide layer. Due to the angle of incidence of the beam on the sample (approximately $14^{\circ}$ ), the $0.2 \times 0.2 \mu \mathrm{m}$ focused beam spot was distorted to roughly $0.2 \times 1 \mu \mathrm{m}$, with the long axis aligned parallel to the oxide-metal interface. The step size used was $0.2 \mu \mathrm{m}$. The energy of the x-ray beam was $17.79 \mathrm{keV}$, corresponding to a wavelength of $0.6969 \AA$. The scanning process and the types of data collected are illustrated schematically in Figure 3.

Elemental fluorescence information was used primarily to determine the location of the x-ray beam spot during the experiment, but was also useful for identifying and confirming characteristics such as the oxide thickness, oxide-metal interface location, and second-phase precipitate location. Additionally, any significant element 
redistribution caused by the corrosion process could be qualitatively studied using this technique. Figure 4 shows an example plot of zirconium, tin, and iron fluorescence counts from sample L1581, which confirms the oxide thickness calculated through weight gain of approximately $40 \mu \mathrm{m}$, and shows the location of the oxide-metal interface through a sharp increase in $\mathrm{Zr}$ counts. The presence and location of iron-chrome second phase precipitates can be identified by localized ( $1 \mu \mathrm{m}$ or less) simultaneous spikes in the iron and chrome fluorescence counts. While the precipitates are on the order of the beam size, the beam has a certain interaction volume with the sample that causes them to appear larger. The tin is seen to remain in solid solution in both the metal and oxide with a small but discernible drop in signal within the oxide layer.

To quantify the diffraction information it was necessary to perform an integration of the diffracted intensities recorded on each 2-D diffraction pattern. The integration process is illustrated schematically on the right side of Figure 3. Each diffraction pattern is integrated around the circumference of the partial diffraction ring (spanning a Chi angle of roughly $35^{\circ}$ ) to create a plot of diffracted intensity versus two-theta angle. The integration area is set as a wedge-shape that maintains a constant chi-angle with increasing two-theta such that each diffraction ring is integrated over an equal fraction of the overall circumference. After integration the values obtained for each two-theta angle are corrected for Lorenz polarization before they are analyzed as described in [18].



Figure 3 - Schematic drawing showing the experimental geometry and the types of data acquired at the 2-ID-D synchrotron beamline. 


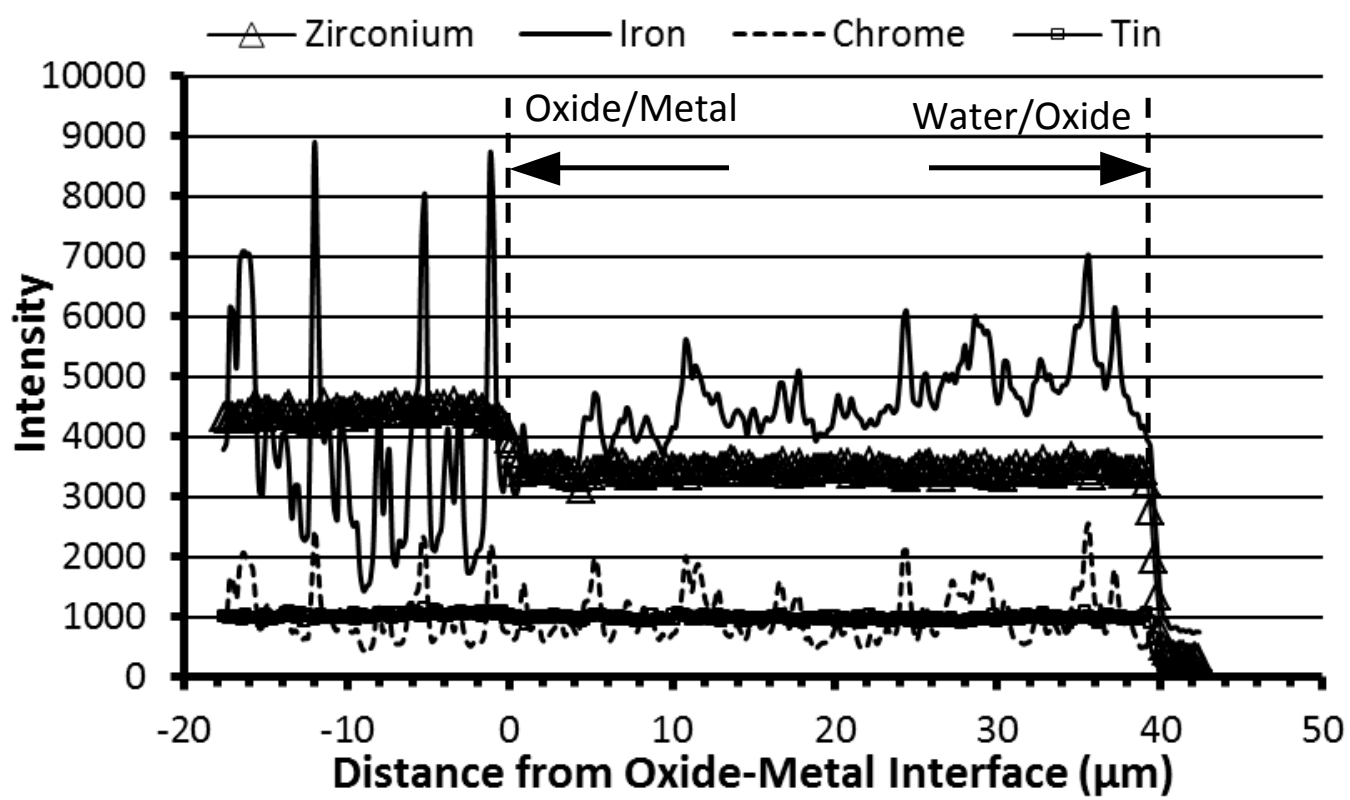

Figure 4 - X-ray fluorescence scan of $Z r$ and alloying elements ${ }^{1}$ in Zircaloy-4 sample $\mathrm{L} 1581$, exposed to $360^{\circ} \mathrm{C}$ water for 1260 days.

${ }^{1}$ The intensity value is a product of the fluorescence line examined. The Fe intensity is higher than the $\mathrm{Zr}$ intensity because the Fe was measured using a K-line, compared to the $\mathrm{Zr}$ L-line.

After each diffraction pattern was integrated, the diffracted intensity curves (also referred to as intensity vs. two-theta curves, see Figure 3 ) generated were deconvoluted and indexed in order to identify the phase composition of the oxide layer.

Peak deconvolution was accomplished using an AISN Software Inc. program called PeakFit [19]. This program allows the user to reproduce an experimental diffracted intensity curve by inserting peaks where necessary and modifying the shape, size, location, and number of peaks to fit the experimental spectrum as closely as possible.

The fitting process for each experimental curve encompassed two basic steps, background removal and curve fitting. The background was removed using a largely visual estimate along with the PeakFit background removal tool, which used a best fit model (meaning whichever model fit best, exponential, quadratic, logarithmic, etc.) to determine the overall shape of the background curve.

Following the initial fit an iterative process was used to modify the peaks by comparing them to powder diffraction. Peaks from a number of different phases were possible, including hcp $\mathrm{Zr}$ (pdf\# 05-0665), monoclinic $\mathrm{ZrO}_{2}$ (pdf\# 37-1484), tetragonal $\mathrm{ZrO}_{2}$ (pdf\# 42-1164)), and $\mathrm{Zr}$ delta hydride (pdf\# 34-0649) as well as $\mathrm{Zr}_{3} \mathrm{O}$ suboxide in the twotheta range studied. $R^{2}$ values were typically above $99 \%$, with no systematic errors in the peak residuals. Table 2 contains a summary of the lattice parameters of the expected crystalline phases in Zircaloy- 4 metal and oxide. 
Table 2 - Lattice parameters of the crystalline phases expected to be observed in Zircaloy-4 metal and oxide layers.

\begin{tabular}{ccccccccc}
\hline & System & $\mathbf{a}(\mathbf{n m})$ & $\mathbf{b}(\mathbf{n m})$ & $\mathbf{c}(\mathbf{n m})$ & $\boldsymbol{\alpha}$ & $\boldsymbol{\beta}$ & $\boldsymbol{\gamma}$ & PDF \# \\
\hline $\mathrm{ZrO}_{2}$ & Monoclinic & 0.53129 & 0.52125 & 0.51471 & $90^{\circ}$ & $99.218^{\circ}$ & $90^{\circ}$ & $37-1484^{1}$ \\
$\mathrm{ZrO}_{2}$ & Tetragonal & 0.364 & - & 0.527 & $90^{\circ}$ & $90^{\circ}$ & $90^{\circ}$ & $42-1164$ \\
$\alpha-\mathrm{Zr}$ & Hexagonal & 0.3232 & - & 0.5147 & $120^{\circ}$ & $90^{\circ}$ & $90^{\circ}$ & $05-0665$ \\
$\mathrm{Zr}_{3} \mathrm{O}$ & Hexagonal & 0.5563 & - & 0.31185 & $120^{\circ}$ & $90^{\circ}$ & $90^{\circ}$ & $22-1025$ \\
$\delta-\mathrm{ZrH}_{1.66}$ & Cubic & 0.4781 & - & - & $90^{\circ}$ & $90^{\circ}$ & $90^{\circ}$ & $34-0649$ \\
\hline
\end{tabular}

${ }^{1}$ Note that this this diffraction card describes the monoclinic phase such that the (200) plane has the lowest two-theta value of the $\{200\}$ family, which is reversed from some older card files.

\section{Results and Discussion}

\subsection{Microdiffraction Phase Analysis}

The peak center locations observed during the fitting process the primary phases present in the oxide layer to be identified as monoclinic and tetragonal $\mathrm{ZrO}_{2}$. To ensure that each diffraction peak was associated with the appropriate phase, the observed experimental peak location was compared to the ideal peak location calculated in the PDF. Studies of this type have previously examined oxides grown on similar alloys under different corrosion conditions. Results in this section are presented as an investigation of samples specific to this study as well as for comparison and validation against previous work.

An example of the comparison of the experimental and PDF two-theta peak locations is shown in Table 3. The data is taken from two representative locations in sample L1581, one in the bulk oxide $12 \mu \mathrm{m}$ from the oxide-metal interface and the second in the metal $1.4 \mu \mathrm{m}$ ahead of the oxide-metal interface. In this instance the difference in peak center two-theta between the observed and indexed peaks is well below 1 percent, with the exception of the (101) tetragonal $\mathrm{ZrO}_{2}$ peak, which was found systematically at a higher two-theta than the PDF value. However, a systematic shift of the (101)T peak such as seen here has been observed in previous studies $[18,20]$. The level of agreement between experimental and PDF peak center locations shown in Table 3 was observed throughout the oxide layers studied. This level of agreement was considered acceptable and indicative of proper identification of the diffraction peaks. 
Table 3 - Representative comparison of experimentally observed peak locations to literature values for sample L1581.

\begin{tabular}{|c|c|c|c|c|c|}
\hline Phase & (h k l) & d-spacing (A) & Calculated $2 \theta$ & Observed $2 \theta^{1}$ & $\% \Delta$ \\
\hline Monoclinic $\mathrm{ZrO}_{2}$ & $\left(\begin{array}{lll}0 & 0 & 1\end{array}\right)$ & 5.0870 & 7.85581 & 7.86747 & 0.148 \\
\hline Monoclinic $\mathrm{ZrO}_{2}$ & $\left(\begin{array}{lll}1 & 1 & 0\end{array}\right)$ & 3.6980 & 10.8141 & 10.8229 & 0.081 \\
\hline Monoclinic $\mathrm{ZrO}_{2}$ & $\left(\begin{array}{lll}0 & 1 & 1\end{array}\right)$ & 3.6390 & 10.9900 & 10.9982 & 0.075 \\
\hline Monoclinic $\mathrm{ZrO}_{2}$ & $\left(\begin{array}{lll}-1 & 1 & 1\end{array}\right)$ & 3.1650 & 12.6421 & 12.6642 & 0.175 \\
\hline Tetragonal $\mathrm{ZrO}_{2}$ & $\left(\begin{array}{lll}1 & 0 & 1\end{array}\right)$ & 2.9950 & 13.3629 & 13.5620 & 1.49 \\
\hline Monoclinic $\mathrm{ZrO}_{2}$ & $\left(\begin{array}{lll}1 & 1 & 1\end{array}\right)$ & 2.8410 & 14.0908 & 14.0938 & 0.021 \\
\hline $\mathrm{Zr}_{3} \mathrm{O}$ & $\left(\begin{array}{lll}1 & 1 & 0\end{array}\right)$ & 2.8170 & 14.2115 & 14.2102 & 0.009 \\
\hline$\alpha-Z r$ & $\left(\begin{array}{lll}1 & 0 & 0\end{array}\right)$ & 2.7980 & 14.3085 & 14.3005 & 0.056 \\
\hline$\delta-\mathrm{ZrH}_{1.66}$ & $\left(\begin{array}{lll}1 & 1 & 1\end{array}\right)$ & 2.7608 & 14.5084 & 14.5513 & 0.295 \\
\hline Tetragonal $\mathrm{ZrO}_{2}$ & $\left(\begin{array}{lll}0 & 0 & 2\end{array}\right)$ & 2.6350 & 15.1987 & 15.2399 & 0.271 \\
\hline Monoclinic $\mathrm{ZrO}_{2}$ & $\left(\begin{array}{lll}0 & 2 & 0\end{array}\right)$ & 2.6060 & 15.3688 & 15.4036 & 0.226 \\
\hline$\alpha-Z r$ & $\left(\begin{array}{lll}0 & 0 & 2\end{array}\right)$ & 2.5730 & 15.5672 & 15.5766 & 0.061 \\
\hline Monoclinic $\mathrm{ZrO}_{2}$ & $\left(\begin{array}{lll}0 & 0 & 2\end{array}\right)$ & 2.5400 & 15.7707 & 15.7649 & 0.037 \\
\hline $\mathrm{Zr}_{3} \mathrm{O}$ & $\left(\begin{array}{lll}1 & 1 & 6\end{array}\right)$ & 2.4770 & 16.1744 & 16.1947 & 0.125 \\
\hline$\alpha-Z r$ & $\left(\begin{array}{lll}1 & 0 & 1\end{array}\right)$ & 2.4590 & 16.2936 & 16.3085 & 0.091 \\
\hline Monoclinic $\mathrm{ZrO}_{2}$ & $\left(\begin{array}{lll}1 & 0 & 2\end{array}\right)$ & 2.3340 & 17.1727 & 17.2079 & 0.205 \\
\hline Monoclinic $\mathrm{ZrO}_{2}$ & $\left(\begin{array}{lll}0 & 1 & 2\end{array}\right)$ & 2.2845 & 17.5477 & 17.5625 & 0.084 \\
\hline
\end{tabular}

${ }^{T}$ The observed values shown are from two representative single locations, one in the oxide and one in the metal. The observed center location can shift slightly, with standard deviations for the three phases of $\quad 2=0.0047$, $2=0.0103$, and $\quad=0.0022$ (all in degrees two-theta). A single standard deviation for the monoclinic phase center locations was calculated by averaging the $\sigma$ value at each point as calculated by PeakFit over the entire oxide, then averaging all of the monoclinic peaks together. The tetragonal and hcp $\mathrm{Zr} \sigma$ values were averaged over one or two peaks in a similar manner.

Figure 5 shows the diffracted intensity versus two-theta plotted in both a 3-D surface format and as a contour plot for a full scan of the beam across the oxide layer and into the metal in sample N2511. A number of oxide and metal peaks can be identified which correspond to the monoclinic and tetragonal phases, as well as the hexagonal closepacked (HCP) $\mathrm{Zr}$ metal. Peaks associated with the monoclinic $\mathrm{ZrO}_{2}$ phase are the primary peaks visible within the oxide layer, with only one tetragonal $\mathrm{ZrO}_{2}$ peak (the $(101)_{T}$ ) clearly visible. A second tetragonal peak (the $(002)_{T}$ ) was identified in the diffraction patterns but is not easily visible in this figure. The metal shows two major peaks corresponding to the HCP structure. A third metal peak, the $(0002)_{\mathrm{zr}}$, is present only at low levels. It should be noted that the small spot size of the x-ray beam $(0.2 \mu \mathrm{m})$ compared to the grain size of the Zircaloy substrate $(\sim 20 \mu \mathrm{m})$ means that the diffraction 
data obtained from the metal likely comes from a single grain. A delta- $\mathrm{ZrH}_{2}$ hydride peak can also be seen alongside the metal peaks in certain locations (since the hydrides are thin platelets in cross-section they are not expected to appear continuously). The phases observed are consistent with those seen in previous studies of $\mathrm{Zr}$ alloy oxides [12, 16].

A marked periodic variation in intensity can be observed in several of the oxide peaks throughout the oxide thickness. The periodic variations correspond approximately to the distance between oxide transitions. The distance between the periods in Figure 5 is $1.9 \mu \mathrm{m}$ on average, which is in good agreement of the transition thickness of Zircaloy-4 $(1.8 \mu \mathrm{m})$ observed in previous studies $[13,15,16]$.

Qualitative observations on the oxide and metal texture can be made by noting the relative intensity of the diffraction peaks. The oxide shows strong $(111)_{\mathrm{M}}$ and $(-111)_{\mathrm{M}}$ peaks and a number of less intense monoclinic peaks. The $(111)_{M}$ and $(-111)_{M}$ peaks are expected to show the largest intensity since the texture of the oxide multiplies their diffracted intensity over more strongly aligned but less numerous planes such as the $\{200\}$ family of planes. Notably missing is the $(200)_{M}$, while the $(020)_{M}$ and $(002)_{M}$ peaks show strong intensities. The observed peaks are consistent with the (200) planes being roughly parallel to the plane of the oxide-metal interface, as observed in previous studies [21]. Based on our experimental geometry, the oxide grains developed with their $(200)_{M}$ planes nearly parallel to the global oxide-metal interface. In the metal three strong peaks can be seen, two from HCP Zr and one from delta-hydride. The (0002) HCP basal plane peak is barely visible when present at all. This observation is consistent with the sample having the rolling direction aligned with the cross-sectional sample surface normal direction: as based on the texture of rolled sheet Zircaloy-4 the basal poles can be expected to be primarily oriented in the normal-transverse plane.

Another notable feature is the consistency in the diffracted intensity of the oxide peaks between transitions. Each peak appears to return to roughly the same level of intensity after each transition, which suggests that the oxide develops with an identical structure and texture through every transition, and that a repetitive mechanism is operative during oxide formation.

The peak intensities shown in Figure 5 do not represent of a single angle, but are rather an average over the integrated range. This results in some loss of orientation information, and makes it difficult to determine the exact alignment of a given plane. However the data presented here shows general agreement with the expected texture of Zircaloy-4 oxide and metal. 


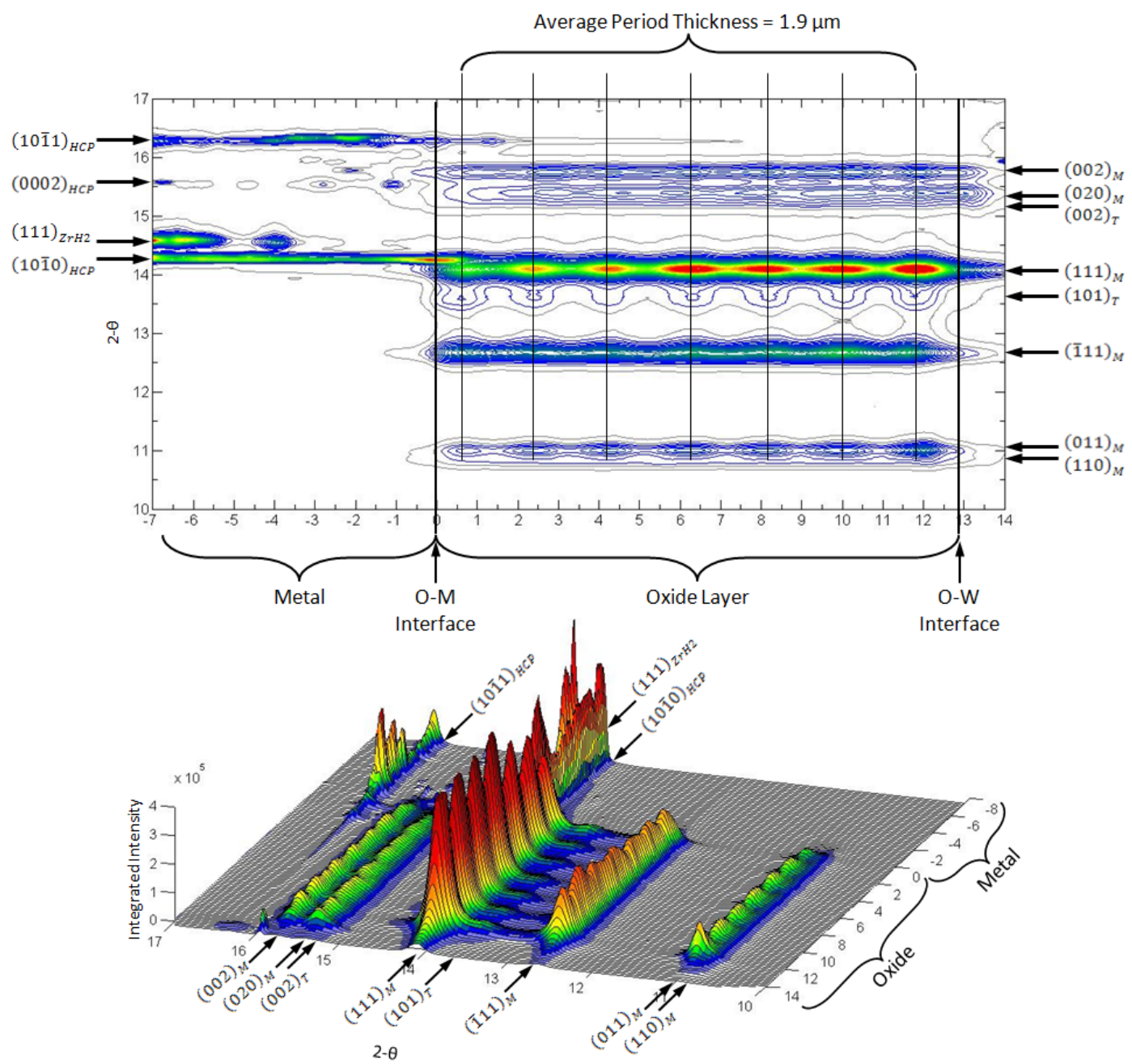

Figure 5 - Surface and contour maps of the diffracted x-ray intensity versus degrees two-theta angle scans from the oxide-water interface into the metal substrate showing the diffraction peaks originating from the Zircaloy-4 oxide layer and base metal in sample N2511. The subscripts ' $M$ ' and ' $T$ ' stand for monoclinic and tetragonal oxide peaks, 'HCP' for zirconium metal peaks, and 'ZrH2' for hydride peaks.

The x-ray diffracted intensity versus two-theta angle for a full oxide scan is shown for sample L1581 in Figure 6. Similar observations can be made of the oxide and metal peaks as those seen in Figure 5; however the details are less evident due to the much larger thickness of oxide. Monoclinic and tetragonal oxide peaks are visible alongside $\mathrm{Zr}$ metal and hydride peaks. Periodic intensity variation of the oxide peaks in Figure 6 is not as visible to the same degree as visible in Figure 5, even though four clear oxide transitions are visible in the corrosion kinetics for L1581 shown in Figure 1. As will be shown later, a similar region of periodicity is found between 30 and 40 micron from the oxide-metal interface. This suggests that for thicker oxides, the regions of cyclic oxide cracks may only last for 10 to 20 microns before the cycles become disrupted. This is consistent with the cross-sectional oxide images shown in Figure 2. 

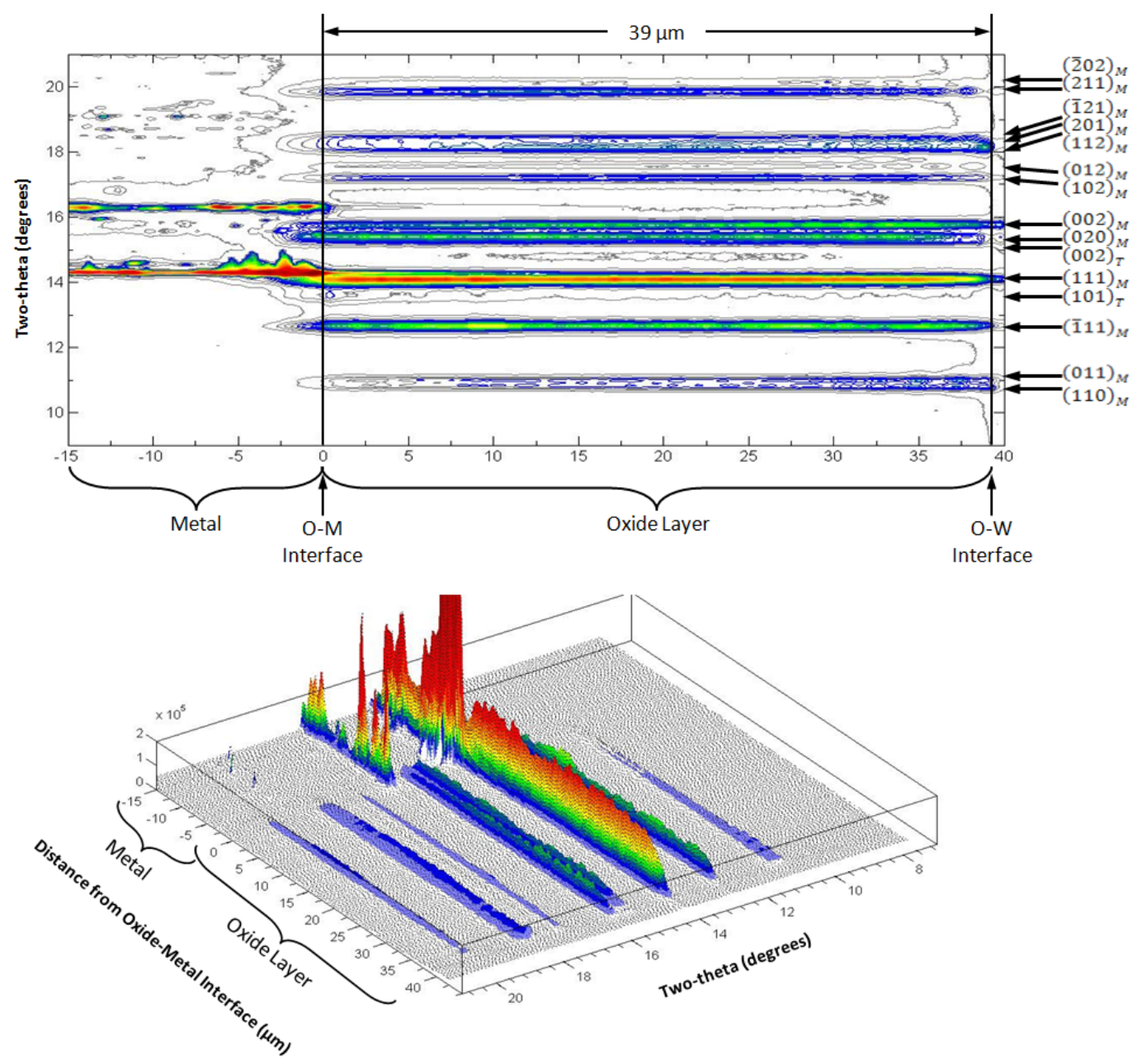

Figure 6 - Surface and contour maps of the diffracted x-ray intensity versus degrees two-theta angle scans from the oxide-water interface into the metal substrate showing the diffraction peaks originating from the Zircaloy-4 oxide layer and base metal in sample L1581. The subscripts ' $M$ ' and ' $T$ ' stand for monoclinic and tetragonal oxide peaks, 'HCP' for zirconium metal peaks, and 'ZrH2' for hydride peaks.

Figure 7 presents the variation in diffracted intensity for several of the primary monoclinic peaks as a function of position in the oxide layer with the intensity plotted as the integrated area under the diffraction peak. A number of observations can be made about the oxides grown on samples N2511 and L1581 using the curves presented in Figure 7. Figure 7(A) corresponds to data from sample N2511 and Figure 7(B) to data from sample L1581. Note that the focused $0.2 \mu \mathrm{m}$ x-ray beam experiences a small spreading effect due to its incident angle on the sample surface, and has an interaction volume internal to the sample. This is the cause of the non-zero intensity entry and exit profiles for the oxide peak intensities in the figure.

In Figure 7(A), the monoclinic and tetragonal $\mathrm{ZrO}_{2}$ peaks show strong periodic intensity variation throughout the oxide thickness. The peak-to-peak distance can be measured 
at $1.9 \mu \mathrm{m}$, which as mentioned earlier corresponds well to the measured Zircaloy-4 oxide transition thickness. It should be noted that multiple scans across N2511 were made, and that the scan shown in Figure $7(\mathrm{~A})$ exhibited the clearest periodicity through the entire oxide thickness.

The diffraction peaks in Figure 7(A) appear to show a relationship with each other; the intensity of many of the peaks is aligned and periodically goes up and down at the same time This could indicate that periodic variations in diffracted intensity are not a result of changes in the oxide phase content, but are rather a result of cracking, porosity, or some other physical variation that periodically reduces the overall diffracted intensity. Previous findings [13] showed that intensity variations between the $(020)_{M}$ and $(101)_{T}$ displayed the opposite behavior. Some peaks were observed to show little periodic variation, as the $(-111)_{\mathrm{M}}$ peak illustrates. Furthermore, the $(012)_{\mathrm{M}}$ appears misaligned with the rest of the monoclinic peaks, suggesting that in the regions of low signal (presumably in the cracked regions), that a different oxide texture is present.

Periodic intensity variation can be seen again in Figure 7(B) for sample L1581. However, significantly less clear periodicity is visible compared to that seen in Figure $7(\mathrm{~A})$ for N2511. The periodicity of the $(011)_{\mathrm{M}}$ peak is the most well defined, but is only evident in the outermost 6-8 microns of oxide formed, which agrees well with the three transitions visible in the corrosion kinetics for L1581 shown in Figure 2. The loss of periodicity could be attributed to the transition to linear corrosion kinetics, or is more likely due to the path of the x-ray beam across the oxide. As shown in Figure 2, it is difficult to find a path through the oxide that will trace over a region of oxide cracks for more than 10 microns or so at a time.

While not shown in Figure 7, analysis showed the $(200)_{M}$ peak intensity to be very low or non-existent in both $\mathrm{N} 2511$ and L1581. The $(020)_{M}$ and $(002)_{M}$ peaks were clearly visible. We can arrive at a similar conclusion as previously stated that the oxide grains grow in an orientation such that their $(200)_{M}$ plane is more-or-less aligned parallel to the oxide-metal interface. Previous studies have shown that oxides grown on other $\mathrm{Zr}$ alloys maintain a similar grain orientation [13].

In addition to the $(101)_{T}$ peak, a second tetragonal peak identified as the $(002)_{T}$ peak was also observed in both N2511 and L1581 throughout the oxide thickness. This peak had previously been observed near the oxide-metal interface in Zircaloy-4, ZIRLO ${ }^{\circledR}$, and model $\mathrm{Zr}$ alloys [15], and was postulated as an indicator for a precursor phase of monoclinic $\mathrm{ZrO}_{2}$. However, the $(002)_{\mathrm{T}}$ peak intensity did not show any periodic trends or relationships with other peaks and was seen throughout the thickness of the oxide in this study. This observation does not support the idea that the $(002)_{T}$ peak indicates a monoclinic precursor phase. The $(002)_{T}$ peak did not show an increase in intensity near the oxide-metal interface as observed for the $(101)_{T}$ peak. 



Figure 7 - Variation of the diffracted intensity as a function of distance from the oxidemetal interface for the most prominent oxide peaks in samples (A) N2511 and (B) L1581.

\subsection{Tetragonal Fraction}

The relationship between the tetragonal and monoclinic $\mathrm{ZrO}_{2}$ phases can be quantified by calculating the tetragonal fraction $f_{T}$ using the well-known Garvie-Nicholson formula, as shown below in Equation 3.1 [22].

$$
=101111+101+111
$$

where 101 is the integrated intensity under the (101) tetragonal peak and 111 and

111 correspond to the integrated intensities of the (111) and (-111) monoclinic peaks respectively. Note that the tetragonal fraction as presented in Equation 3.1 is uncorrected for the monoclinic and tetragonal oxide textures. The standard GarvieNicholson formula is strictly valid for powders or other random texture materials. In principle, peak intensities should be corrected for texture to be quantitatively correct, 
which requires a pole figure for each sample. Given a pole figure, intensity correction factors can be calculated for each peak intensity based on individualized texture.

Texture correction factors were calculated by Yilmazbayhan et al. in [12] for zirconium alloys, where a more detailed explanation of the texture correction process can also be found.

Figure 8 presents the uncorrected tetragonal fraction as calculated for N2511 and L1581. The maximum values of $f_{T}$ can be seen to be approximately $15 \%$ in $\mathrm{N} 2511$ and $9 \%$ in L1581. The peak $f_{T}$ occurs at the oxide-metal interface in both samples, and steadily decreases over a distance of 2-3 $\mu \mathrm{m}$ to a stable average value in the bulk oxide in agreement with previous results [12]. The average bulk oxide tetragonal fraction is 5$6 \%$ in N2511 and roughly 3\% in L1581. Texture corrections using the factors determined by Yilmazbayhan et al. were performed, but are not presented. It was found that the texture correction reduces the overall tetragonal fraction but does not drastically change the shape or trends of the curve. In Figure 8(A), the shape of the tetragonal fraction curve follows that of the three Garvie-Nicholson peak intensity curves, since all of the peaks in the formula are periodic and aligned with each other.

The calculated tetragonal fraction in both samples agrees well with the results found in previous studies $[12,20]$ of Zircaloy- 4 , which found that the uncorrected tetragonal fraction was systematically highest at the oxide-metal interface with a value of $10-15 \%$, decreasing to less than $10 \%$ at distances $>3 \mu \mathrm{m}$ from the oxide-metal interface. 


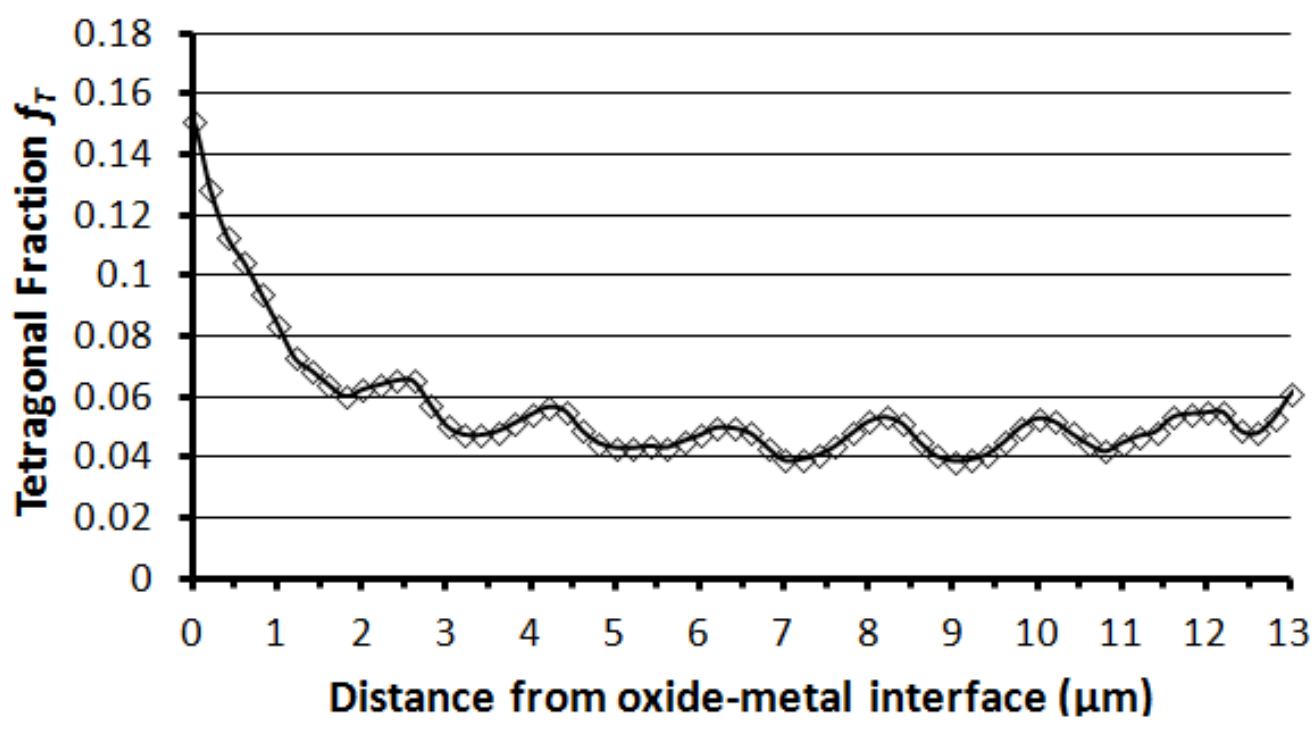

$\neg$ L1581

B

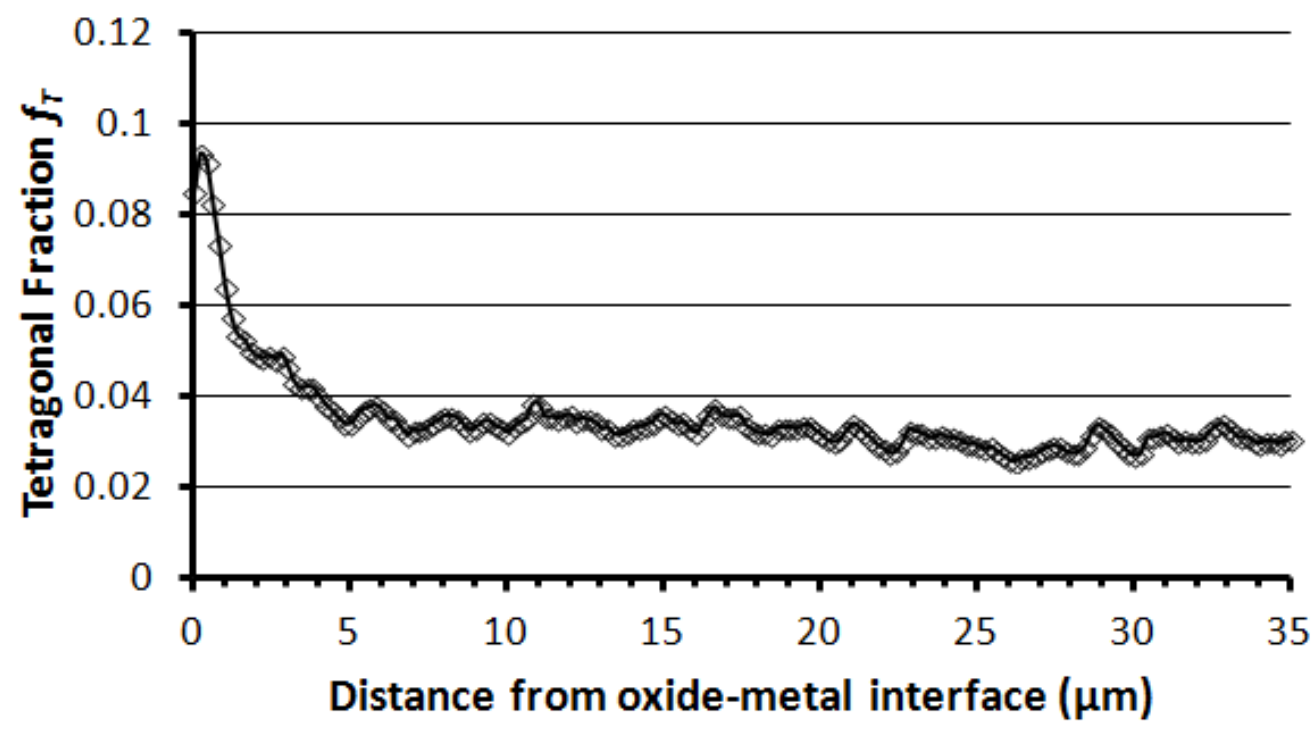

Figure 8 - Tetragonal fraction as a function of distance from the oxide-metal interface for the oxide layers grown on (A) N2511 and (B) L1581 calculated using the uncorrected Garvie-Nicholson formula, as described in the text.

Observations of the spatially resolved monoclinic and tetragonal phase diffracted intensity and the tetragonal fraction support the hypothesis that the oxide forms in discrete layers by periodically nucleating new grains followed by a period of grain growth. The periodic variations in the diffracted intensity suggests a cyclic growth mechanism and the high tetragonal fraction at the oxide-metal interface suggests that tetragonal grains are nucleated at the interface before transforming into monoclinic grains during continued growth. 


\subsection{Grain Size}

In addition to peak area, Full Width Half Maximum (FWHM) information was extracted from the fitted peak summaries. The peak FWHM can be used to determine grain size (in the direction normal to the diffracting plane) as long as the size is less than $\sim 200 \mathrm{~nm}$, although, strain within the grain can also cause peak broadening. Note that for this application the term 'grain size' actually refers to the grain column diameter, since the diffracting planes measured span the oxide column width rather than the length. Application of the FWHM to calculate grain size has been accomplished by previous studies, but this work includes observations of the strain near the oxide-metal interface to support conclusions on oxide growth mechanisms.

Figure 9 shows the FWHM for several of the main monoclinic and tetragonal peaks as a function of position in the oxide layer, with error bars representing the average standard deviation for the monoclinic and tetragonal phases. The average bulk oxide FWHM for the monoclinic peaks is approximately 0.2 degrees two-theta, while the average for the $(101)_{T}$ is approximately 0.38 degrees two-theta. The FWHM for the tetragonal phase is expected to be larger than that for the monoclinic phase because of the much smaller tetragonal grain size [14].

The monoclinic peaks appear to maintain a relatively constant FWHM through the thickness of the bulk oxide in both samples. However, an increase in the monoclinic FWHM can be seen starting at a distance of 2-3 $\mu \mathrm{m}$ from the oxide-metal interface and reaching a maximum at the interface. Most notable is the increase in the $(020)_{M}$ which

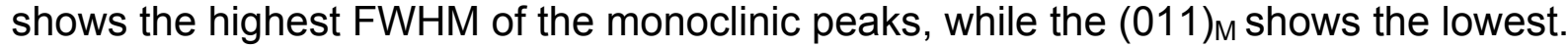
The tetragonal FWHM appears to be relatively constant in L1581 (see Figure 9B), but shows a decrease that mirrors the monoclinic (020) FWHM increase near the oxidemetal interface.

Both the monoclinic and tetragonal phases show a change in their peak FWHM near the oxide-metal interface; the monoclinic FWHM gets larger and the tetragonal FWHM gets smaller. Near the interface, both monoclinic and tetragonal phase grains are growing. As they develop, the monoclinic grains increase in size and the larger tetragonal grains transform into the monoclinic phase. As the large tetragonal grains transform and leave only the smaller stress-stabilized tetragonal grains behind, the tetragonal FWHM increases as the average tetragonal grain size decreases. The opposite is true for the monoclinic phase; the FWHM gets smaller as the monoclinic grains grow. 


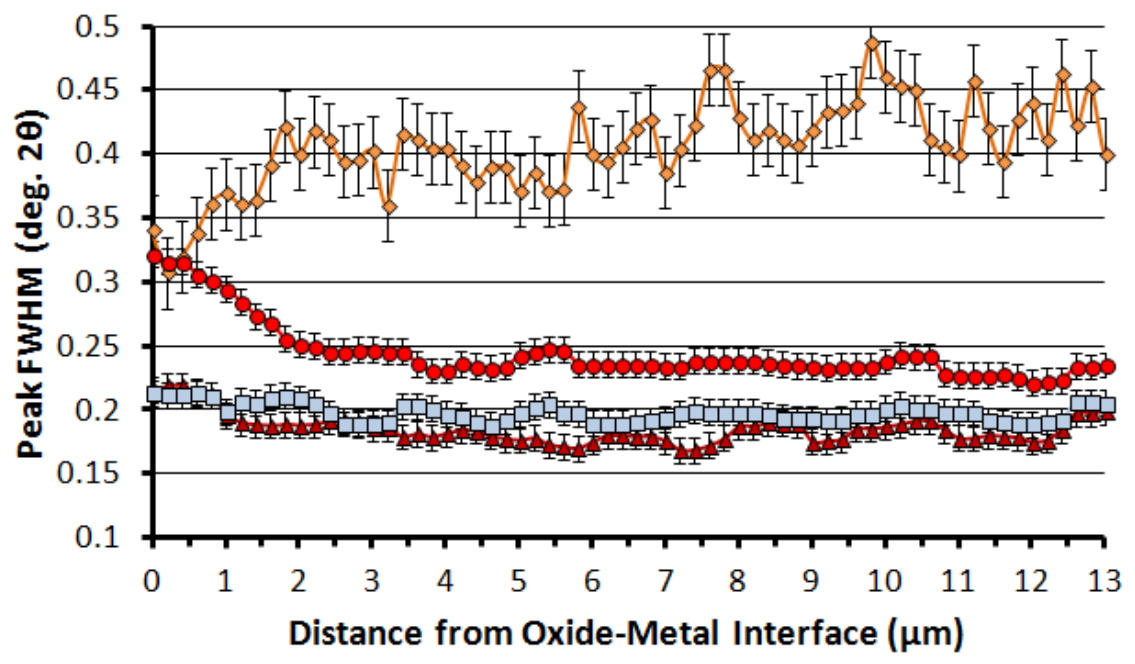

A

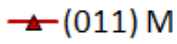

$\neg(101) \mathrm{T}$

$\rightarrow(020) M$

$\square-(002) \mathrm{M}$

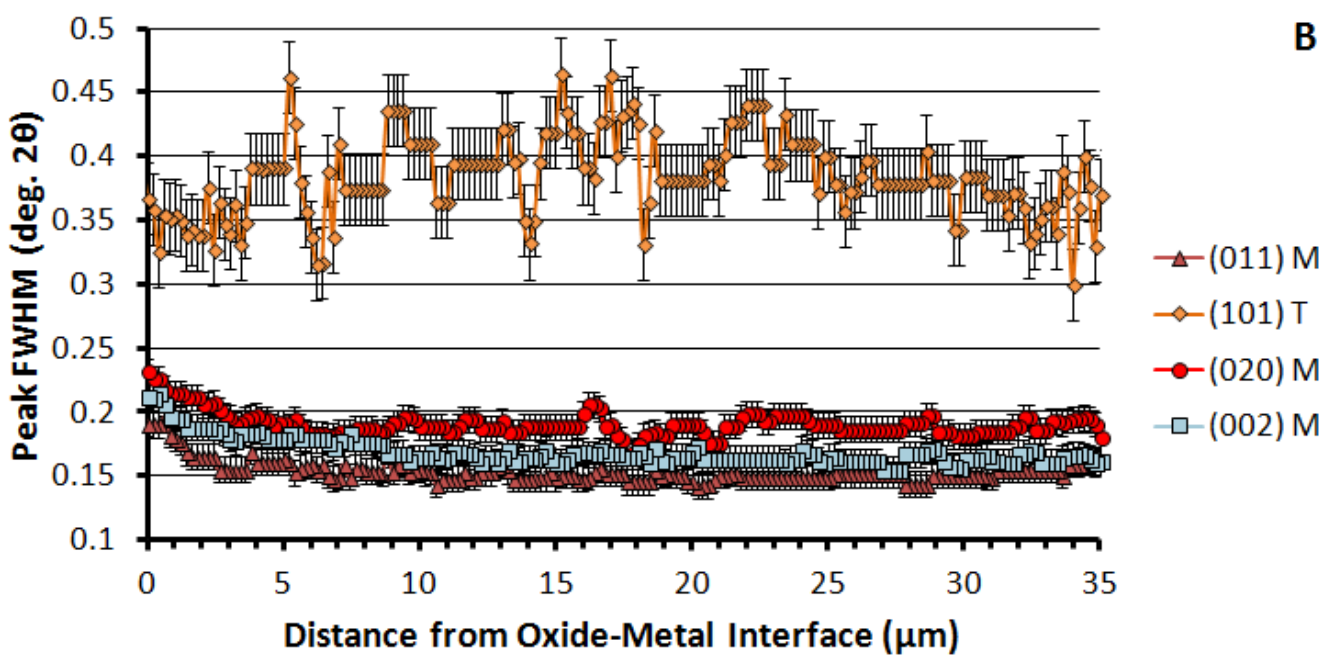

Figure 9 - Peak FWHM shown for some of the primary monoclinic and tetragonal diffraction peaks in samples (A) N2511 and (B) L1581. All monoclinic peaks not shown had a FWHM between that of the $(011)_{\mathrm{M}}$ and the $(020)_{\mathrm{M}}$.

The FWHM was used to calculate the oxide grain size through the Debye-Scherrer equation. The grain size ' $D$ ' is given by the equation as:

$$
=0.9
$$

where $=2-212$ is the grain size broadening for a given peak, $B$ is the measured peak FWHM in radians, $B_{\mathrm{i}}$ is the instrumental broadening measured using a standard, $\theta$ is the diffraction angle (half the measured $2 \theta$ ), and $\lambda$ is the wavelength of the incident radiation $(0.0693 \mathrm{~nm}$ in this instance). The instrumental broadening was not measured specifically for this experiment, but a value of 0.051 degrees two-theta found during previous work using the same instrument and a NIST 660a LaB $_{6}$ powder standard was used instead [12]. 
The Debye-Scherrer formula is valid for sizes up to $100 \mathrm{~nm}$, and is ideally applied towards randomly oriented spherical or equiaxed grains. The geometric condition is not met by the majority of the oxide grains; most of the oxide grains are long and columnar, with a minority of the monoclinic grains and most of the tetragonal grains being small and equiaxed [14]. Given the geometry of the experimental setup, the diffracted beams were primarily from planes oriented perpendicular to the long axis of the columnar grains. Therefore, as stated above, for the monoclinic phase the 'grain size' is representative of the column width rather than the overall grain size. For tetragonal grains, both the size and the equiaxed assumption can be considered valid.

Table 4 shows the average grain size in the bulk oxide calculated using the DebyeScherrer formula for several of the main monoclinic peaks and the (101) tetragonal peak in the bulk oxide. The average monoclinic grain size calculated using the monoclinic peaks shown is $18.6 \mathrm{~nm}$ for $\mathrm{N} 2511$ and $22.7 \mathrm{~nm}$ for L1581. The average tetragonal grain size was found to be approximately $9.4 \mathrm{~nm}$ in both samples. The average grain sizes are consistent with those found for Zircaloy-4 monoclinic and tetragonal phases in similar X-ray diffraction studies as well as TEM observations $[12,15]$.

There is a notable spread in the apparent grain size indicated by the monoclinic peaks examined in both samples. In general it seems the $(020)_{M}$ indicates the smallest grain size and the $(011)_{M}$ the largest, with a difference between the two of $4-5 \mathrm{~nm}$. This is consistent with our FWHM results that show a similar spread.

A significant difference was observed between the calculated monoclinic grain size between N2511 and L1581. The monoclinic grain sizes in L1581 are consistently larger than those in N2511, with a difference of $4-5 \mathrm{~nm}$ or $15-25 \%$ depending on the plane used to make the calculation. Since both samples are Zircaloy-4 of similar composition and fabrication, the most likely reason for the difference in grain size is the corrosion conditions. Sample N2511 was corroded at $316^{\circ} \mathrm{C}$ for 3113 days, while L1581 was corroded at $360{ }^{\circ} \mathrm{C}$ for 1260 days. The higher temperature may have caused L1581 to develop larger oxide grains while the lower temperature and much longer exposure time could have allowed a finer oxide structure to develop on N2511. Interestingly, the tetragonal grain size is similar in both samples at roughly $9.4 \mathrm{~nm}$, perhaps indicating that the tetragonal grains are stabilized only to a certain maximum size at which point they cease to grow or transform into the monoclinic phase [15].

Table 4 - Average bulk oxide grain size calculated using the Debye-Scherrer formula using several of the main monoclinic and tetragonal $\mathrm{ZrO}_{2}$ diffraction peak widths. 


\begin{tabular}{|c|c|c|c|c|}
\hline & \multicolumn{2}{|c|}{ Grain Size $(\mathrm{nm})$} & & \\
\hline Oxide Peak & N2511 & L1581 & Difference $(\mathrm{nm})$ & $\%$ Difference \\
\hline (011) M & 20.7 & 25.1 & 4.5 & 17.8 \\
\hline$(-111) \mathrm{M}$ & 17.9 & 21.6 & 3.7 & 17.1 \\
\hline (111) M & 20.3 & 24.1 & 3.8 & 15.9 \\
\hline (020) M & 15.5 & 19.8 & 4.3 & 21.9 \\
\hline (101) $\mathrm{T}$ & 9.3 & 9.5 & 0.2 & 1.9 \\
\hline
\end{tabular}

Peak broadening near the oxide-metal interface can be attributed to two main causes: strain broadening and grain size [23]. Strain causes the diffraction peaks to broaden due to diffraction from non-uniformly distributed strained planes of a slightly different dspacing from the unstrained planes [23]. Smaller oxide grains result in a greater amount of incoherent $x$-ray scattering than larger grains, which also causes the diffraction peaks to broaden [23]. In this work a Williamson-Hall analysis [24] was used to separate the effects of size and strain broadening on the FWHM.

Size broadening and strain broadening can be expressed according to the following proportions:

$$
\begin{aligned}
& =\quad+ \\
& \alpha \\
& \text { Strain broadening (Eq. 3.3) } \\
& \alpha 0.9 \\
& \text { Size broadening } \\
& \begin{array}{ll}
= & +0.9
\end{array}
\end{aligned}
$$

where $\mathrm{FWHM}_{\text {sample }}$ is the grain size broadening for a given peak (expressed in the same way as for the Debye-Scherrer calculation), $\theta$ is the Bragg angle (in radians), $\varepsilon_{\text {lattice }}$ is the lattice strain, $\lambda$ is the $x$-ray beam wavelength (in $\mathrm{nm}$ ), and $D$ is the grain size (in $\mathrm{nm}$ ).

The method by which a Williamson-Hall plot is used to separate size from strain broadening is shown in Figure 10, where $\left(\mathrm{FWHM}_{\text {sample }} \mathrm{X} \cos \theta\right)$ is plotted against $(\sin \theta)$ for several monoclinic peaks. In such a plot the slope of the plotted curve is proportional to the strain broadening and the $y$-intercept is proportional to the size broadening. A perfectly horizontal line indicates only size broadening, a sloped line with a zero y-intercept indicates pure strain broadening, and a combination of the two indicates the presence of both effects. Both elastic and plastic strains may play a role in peak broadening.

The components of size and strain broadening can be calculated as indicated in Figure 10. The strain component can be obtained from the slope of the line $(C \varepsilon)$, where $C$ is 
some constant and $\varepsilon$ is the strain. The size component is obtained from the $y$-intercept value, $K \lambda / D$, where $K$ is the Scherrer constant (usually assumed as $K=0.9$ ), $\lambda$ is the $x$-ray wavelength, and $\mathrm{D}$ is the grain size.

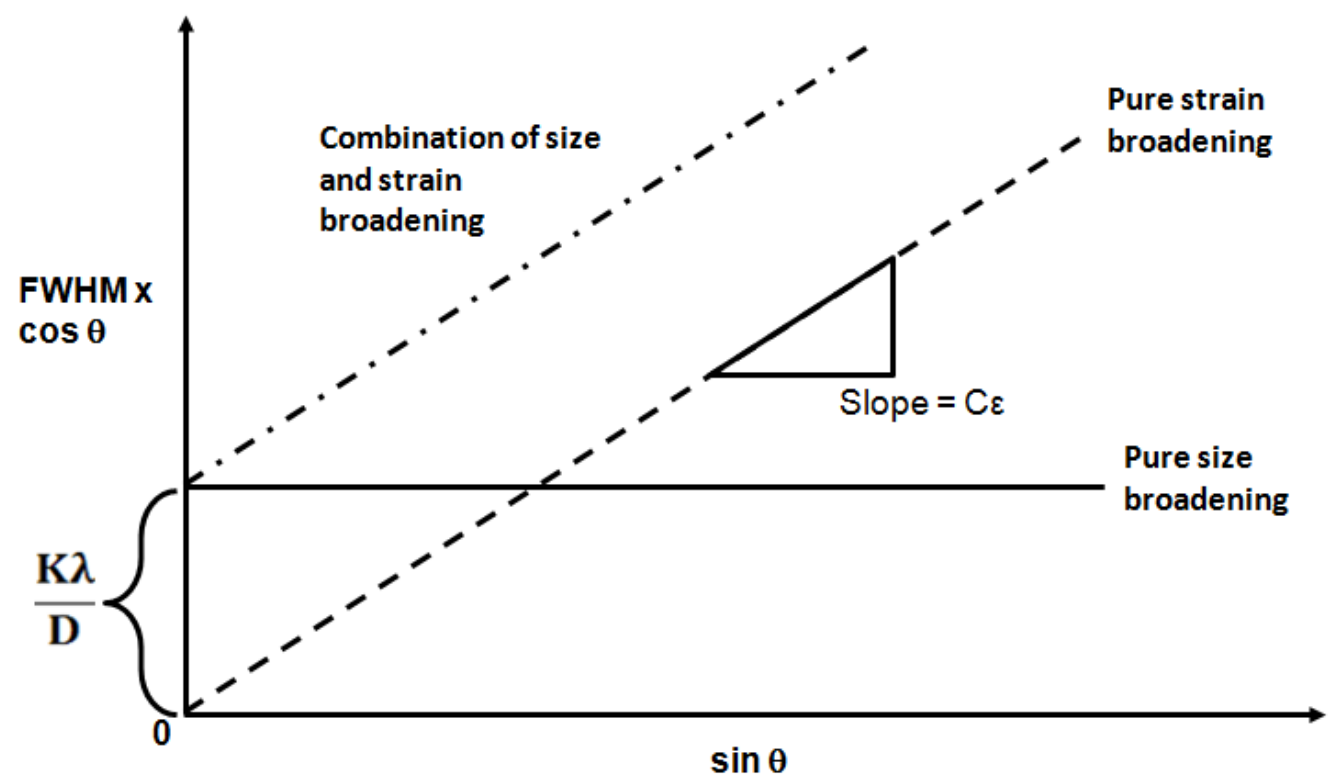

Figure 10 - Schematic of how a Williamson-Hall plot can be used to separate size broadening from strain broadening and how the components of size and strain can be obtained.

Figure 11 shows a Williamson-Hall plot for samples N2511 and L1581 constructed using data from the major monoclinic oxide peaks at various two-theta locations. Data taken from three distinct locations in the oxide are shown; one at the oxide-metal interface, another within one transition distance into the oxide (less than $1.8 \mu \mathrm{m}$ past the oxide-metal interface), and the last within the bulk oxide beyond one transition distance.

In both samples, the slope of the trend lines taken from each location in the oxide remains constant, to a first approximation. It should be noted that small changes in the FWHM, within the error bars as seen in Figure 9, could cause the calculated slopes to vary. Although not every point has been examined, Williamson-Hall plots taken from various other locations were found to closely resemble the representative curves depicted in Figure 11 (the bulk oxide data trends are shown as a solid line). A stress (and therefore elastic strain) gradient in the protective part of the oxide as has been seen by others [3,25,26]; however a few factors could impede this observation. First, the stress present at high temperature is modified during cooling due to differential and anisotropic thermal expansion coefficients between the metal and oxide. Second, the planes studied are parallel to the sample free surface, so that the signal comes from planes that are partially relaxed. Thus the calculated strains should be taken with caution. 


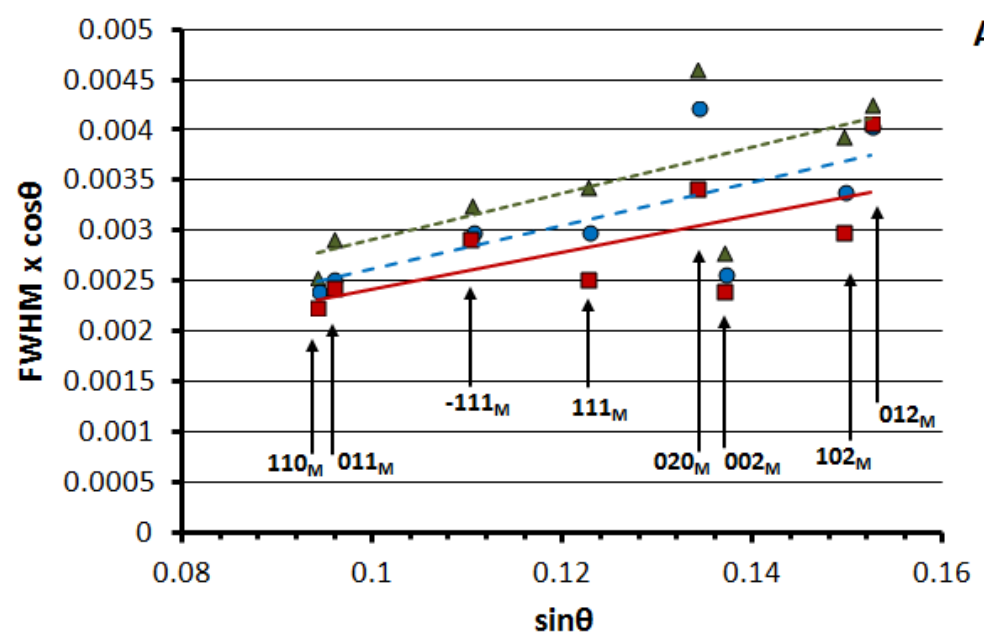

A

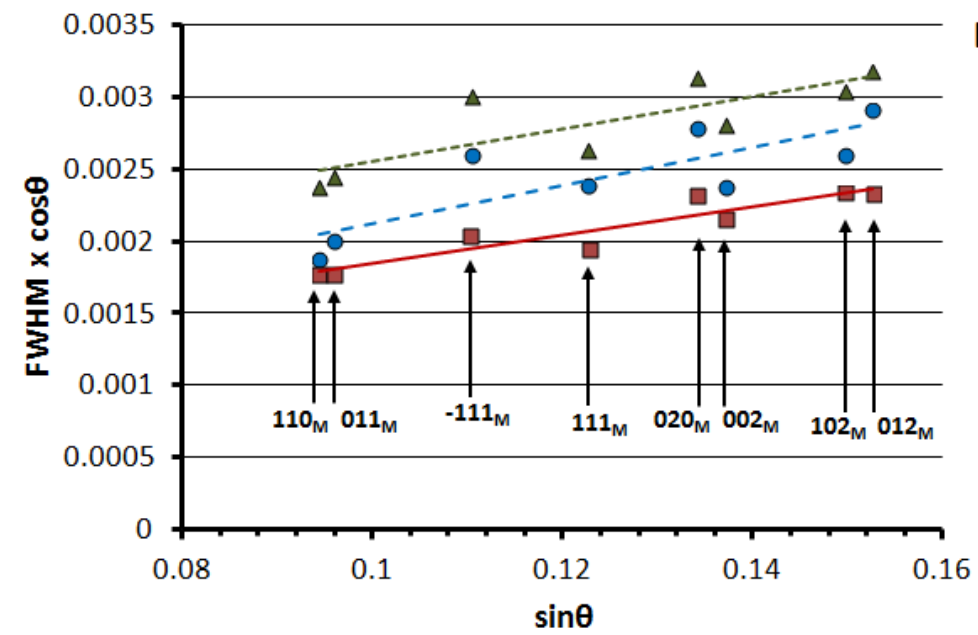

$\Delta \mathrm{O} / \mathrm{M}$ Interface $\mathbf{- - - - - - - - ~}$

- $0.8 \mu \mathrm{m}----$

$\square 2.6 \mu \mathrm{m}$

B

$\Delta \mathrm{O} / \mathrm{M}$ Interface -------

- $1.6 \mu \mathrm{m}----$

$\square 8.6 \mu \mathrm{m}$

Figure 11 - Williamson-Hall plot created using the main observed monoclinic peaks for samples (A) N2511 and (B) L1581 showing the effects of size and strain broadening at various distances into the oxide from the oxide-metal interface. The calculated $\sigma=0.01$ degrees $2 \theta$ does not produce a significant change in the curve shape or alignment.

The $y$-intercept of the fitted lines increases with proximity to the oxide-metal interface, which indicates that the grains are smaller near the oxide-metal interface and suggests that size broadening is the main contributor to the change in peak FWHM near the oxide-metal interface. The monoclinic diffraction peaks are broadest at the interface and become narrower with increasing distance from the interface. From this, we can infer that the monoclinic grains are smallest just for the grains recently created at the oxide-metal interface, increasing in size with residence time in the oxide until reaching a maximum some distance from the interface. This result agrees with current understanding of oxide growth where small grains are nucleated at the oxide-metal interface and the most favorably oriented grains subsequently grow [13]. The increase in the tetragonal phase FWHM with distance from the oxide-metal interface seen in Figure $9(\mathrm{~A})$ indicates that the tetragonal grains are largest at the oxide-metal interface and decrease to a minimum stable size in the bulk oxide or alternatively transform into 
the monoclinic phase. A smaller grain size could aid in phase stability, as previous studies have found that high stress and small grain size help to stabilize the otherwise unstable tetragonal phase [27-29]. Stabilization of larger grains close to the oxide-metal interface could be achieved through stresses at the oxide-metal interface, which may relax some of the oxide.

The grain size was calculated for each oxide location noted in Figure 11 using the size component of the Williamson-Hall plot given as (KND) in Figure 10. This method of calculation automatically excludes the effects of strain broadening. By setting the $y$ intercept of the fit line equal to (KX/D) the grain size 'D' can be found. Table 5 shows the grain sizes calculated for grains at the oxide-metal interface and in the bulk oxide. The grain sizes shown are applicable to the monoclinic phase only. Additionally, given that the monoclinic grains are not equiaxed but are columnar with the major axis close to the [200] direction [14], and the observed peaks originate from planes parallel to the columnar grain sides, the grain size found using this technique represents the width of the columns.

The monoclinic grain size (columnar grain width) was found to be smaller at the oxidemetal interface than in the bulk oxide. Oxide-metal interface grain sizes of roughly 48 $\mathrm{nm}$ and $28 \mathrm{~nm}$ were calculated for N2511 and L1581 respectively. The bulk oxide grain size values of $56.7 \mathrm{~nm}$ for N2511 and $41.6 \mathrm{~nm}$ for L1581 are larger than those found using the Debye-Scherrer formula but still generally agree with previous TEM and microbeam diffraction studies [13-15].

Table 5 - Grain size for oxide grains at the oxide-metal interface and in the bulk oxide calculated using the size component of the Williamson-Hall plot for samples N2511 and L1581.

\begin{tabular}{cccccc}
\hline Sample & $\begin{array}{c}\text { Bulk Oxide } \\
\text { Y-Intercept }\end{array}$ & $\begin{array}{c}\text { O/M Interface } \\
\text { Y-Intercept }\end{array}$ & $\begin{array}{c}\text { Bulk Oxide } \\
\text { Grain Size }(\mathrm{nm})\end{array}$ & $\begin{array}{c}\text { O/M Interface } \\
\text { Grain Size }(\mathrm{nm})\end{array}$ & $\begin{array}{c}\text { Interface/Bulk } \\
\text { Size Ratio }\end{array}$ \\
\hline N2511 & 0.0011 & 0.0013 & 56.7 & 48.0 & 0.846 \\
L1581 & 0.0015 & 0.0022 & 41.6 & 28.4 & 0.682 \\
\hline
\end{tabular}

Unlike the Williamson-Hall method, the Debye-Scherrer formula does not take into account the effect of strain on peak broadening. Since the entire peak broadening is assumed to be a result of size broadening, it can lead to the calculation of a smaller grain size than is actually present if any amount of strain broadening is also present. Our results show that some amount of strain broadening is present in the monoclinic peaks, and as such it may be necessary to adjust the grain size calculated using the Debye-Scherrer formula. While the shortcomings of the Debye-Scherrer method are recognized, we have used data from the present study to perform Debye-Scherrer based grain size calculations for direct comparison with previous work.

Grain size calculations support the hypothesis that after each transition, tetragonal grains nucleate at the oxide-metal interface and then grow to form a new layer of oxide. The monoclinic phase grains were found to be smallest at the oxide-metal interface, supporting the idea that newly formed tetragonal grains may transform into small 
monoclinic grains, which then grow further with residence time in the oxide. WilliamsonHall analyses indicate that the effects of diffraction peak broadening originate from grain growth, and also show that some level of strain is present in the oxide, which is necessary for stabilization of the tetragonal phase.

\section{Conclusions}

A detailed study of oxide layers grown on Zircaloy-4 was performed using synchrotron $\mathrm{x}$-ray diffraction at the Advanced Photon Source at Argonne National Laboratory. The oxide layers were characterized in cross-section using $\mathrm{x}$-ray microdiffraction to determine the oxide microstructure as a function of position in the oxide (phase content, tetragonal fraction, grain size, and orientation relationships). The objective of this study was to discern structural characteristics of the oxide and relate them to oxide growth mechanisms in long exposure oxides. The main conclusions of this study are:

1. Well defined periodic variations in the diffracted intensity of oxide phases as a function of distance from the oxide-metal interface were observed. The average distance between the periods was measured to be $1.9 \mu \mathrm{m}$, which corresponds well with the previously observed oxide transition thickness for Zircaloy-4 in 360 ${ }^{\circ} \mathrm{C}$ water.

2. The oxide layers studied were composed of a mixture of monoclinic and tetragonal $\mathrm{ZrO}_{2}$. Strong periodic relationships were observed between a number of primary monoclinic and tetragonal oxide diffraction peaks, although this observation disagrees with our current theorized oxide growth mechanism and may indicate that geometric variations such as cracks cause periodic diffracted intensity variations rather than oxide structure.

3. Intensity from the $(200)_{M}$ diffraction peak was not observed while the $(020)_{M}$ and $(002)_{M}$ were clearly visible. This is consistent with oxide growth occurring with the $(200)_{M}$ plane of the columnar monoclinic grains aligned parallel to the oxidemetal interface as previously observed.

4. The tetragonal oxide fraction as determined by the Garvie-Nicholson formula was highest at the oxide-metal interface and decreased over a range of approximately $3 \mu \mathrm{m}$ to a stable value in the bulk oxide. The maximum values found at the interface were $15 \%$ and $9 \%$ for two Zircaloy-4 samples, with respective bulk oxide values of $5 \%$ and $3 \%$.

5. The monoclinic oxide diffraction peaks were observed to broaden within 2-3 $\mu \mathrm{m}$ of the oxide-metal interface to a maximum at the interface. The peak broadening was separated using a Williamson-Hall analysis where it was determined that size broadening due to a smaller grain size at the interface was the dominant cause of the broadening.

6. The monoclinic oxide grain size was calculated using the size broadening component of a Williamson-Hall separation of the size and strain broadening. Grain sizes (diameter) of 28 and $48 \mathrm{~nm}$ at the oxide-metal interface and 41 and $56 \mathrm{~nm}$ in the bulk oxide were calculated using this technique. 
7. The grain size (diameter) of the monoclinic and tetragonal oxide was found using the Debye-Scherrer equation. The average monoclinic grain size was found to be $15-20 \mathrm{~nm}$, and the tetragonal somewhat smaller at $9 \mathrm{~nm}$. The monoclinic grain size was observed to be smallest at the oxide-metal interface, growing over a range of 2-3 $\mu \mathrm{m}$ to a stable bulk oxide value. The tetragonal grain size was constant throughout the oxide thickness.

8. A difference in monoclinic oxide grain size of $4-5 \mathrm{~nm}$ was observed in oxides grown at two different temperatures. Oxide grown at a higher temperature over a shorter period of time had a larger monoclinic grain size than oxide corroded at a lower temperature for a longer period of time. This may indicate that corrosion temperature has an influence on the oxide grain size.

\section{Acknowledgements}

Use of the APS was supported by the Department of Energy, Basic Sciences Office, Office of Science under Contract No. W-31-109-Eng-38.

\section{References}

[1] J. Godlewski, "How the Tetragonal Zirconia is Stabilized in the Oxide Scale that is Formed on a Zirconium Alloy Corroded at $400{ }^{\circ} \mathrm{C}$ in steam," presented at the Zirconium in the Nuclear Industry: $10^{\text {th }}$ International Symposium, Toronto, 1994.

[2] J. Godlewski, J. P. Gros, M. Lambertin, J. F. Wadier, and H. Weidinger, "Raman Spectroscopy Study of the Tetragonal-to-Monoclinic Transition in Zirconium Oxide Scales and Determination of Overall Oxygen Diffusion by Nuclear Microanalysis of ${ }^{18} \mathrm{O}, "$ presented at the Zirconium in the Nuclear Industry: $9^{\text {th }}$ International Symposium, Kobe, 1991.

[3] D. H. Bradhurst and P. M. Heuer, "The Influence of Oxide Stress on the Breakaway Oxidation of Zircaloy-2," Journal of Nuclear Materials, vol. 37, pp. 3547, 1970.

[4] J. R. Moon and D. G. Lees, "Cracking in Oxides on Zr Alloys," Corrosion Science, vol. 10, pp. 85-89, 1970.

[5] J. C. Greenbank and S. Harper, "The Mechanism of Breakaway Oxidation in Zirconium Alloys," Electrochemical Technology, vol. 4, pp. 88-92, 1966.

[6] F. Garzarolli, H. Seidel, R. Tricot, and J. P. Gros, "Oxide Growth Mechanism on Zirconium Alloys," presented at the Zirconium in the Nuclear Industry: $9^{\text {th }}$ International Symposium, Kobe, 1991.

[7] P. Barberis and A. Frichet, "Characterization of Zircaloy-4 Oxide Layers by Impedance Spectroscopy," Journal of Nuclear Materials, vol. 273, pp. 182-191, 1999.

[8] P. Bossis, G. Lelievre, P. Barberis, X. Iltis, and F. Lefebvre, "Multi-Scale Characterization of the Metal-Oxide Interface of Zirconium Alloys," presented at the Zirconium in the Nuclear Industry: $12^{\text {th }}$ International Symposium, Toronto, 2000. 
[9] P. Bossis, F. Lefebvre, P. barberis, and A. Galerie, "Corrosion of Zirconium Alloys: Link Between the Metal/Oxide Interface Roughness, the Degredation of the Protective Oxide Layer and the Corrosion Kinetics," Materials Science Forum, vol. 369-372, pp. 255-262, 2001.

[10] B. Cox, "Pore Structure in Oxide Films on Irradiated and Unirradiated Zirconium Alloys," Journal of Nuclear Materials, vol. 148, pp. 332-343, 1987.

[11] B. Cox and Y. Yamaguchi, "The Development of Porosity in Thick Zirconia Films," Journal of Nuclear Materials, vol. 210, pp. 303-317, 1994.

[12] A. Yilmazbayhan, A. T. Motta, R. J. Comstock, G. P. Sabol, B. Lai, and Z. Cai, "Structure of zirconium alloy oxides formed in pure water studied with synchrotron radiation and optical microscopy: relation to corrosion rate," Journal of Nuclear Materials, vol. 324, pp. 6-22, 2004.

[13] A. T. Motta, A. Yilmazbayhan, R. J. Comstock, J. Partezana, G. P. Sabol, B. Lai, and Z. Cai, "Microstructure and Growth Mechanism of Oxide Layers Formed on Zr Alloys Studied with Micro-Beam Synchrotron Radiation," Journal of ASTM International, vol. 2, 2005.

[14] A. Yilmazbayhan, E. Breval, A. T. Motta, and R. J. Comstock, "Transmission electron microscopy examination of oxide layers formed on $\mathrm{Zr}$ alloys," Journal of Nuclear Materials, vol. 349, pp. 265-281, 2006.

[15] A. T. Motta, M. J. G. d. Silva, A. Yilmazbayhan, R. J. Comstock, Z. Cai, and B. Lai, "Microstructural Characterization of Oxides Formed on Model Zr Alloys Using Synchrotron Radiation," Journal of ASTM International, vol. 5, 2008.

[16] J. S. Bryner, "The Cyclic Nature of Corrosion of Zircaloy-4 in 633 K Water," Journal of Nuclear Materials, vol. 82, pp. 84-101, 1979.

[17] E. Hillner, D. G. Franklin, and J. D. Smee, "Long-term corrosion of Zircaloy before and after irradiation," Journal of Nuclear Materials, vol. 278, pp. 334-345, 2000.

[18] A. Yilmazbayhan, "Microstructural Basis of Uniform Corrosion in Zr Alloys," Doctor of Philosophy, Department of Mechanical and Nuclear Engineering, The Pennsylvania State University, University Park, PA, 2004.

[19] "PeakFit," 4.12 ed: Systat Software Inc., 2012.

[20] N. Petigny, P. Barberis, C. Lemaignan, C. Valot, and M. Lallemant, "In situ XRD analysis of the oxide layers formed by oxidation at $743 \mathrm{~K}$ on Zircaloy 4 and $\mathrm{Zr}$ 1NbO," Journal of Nuclear Materials, vol. 280, pp. 318-330, 2000.

[21] H. Li, M. G. Glavicic, and J. A. Szpunar, "A model of texture formation in ZrO2 films," Materials Science and Engineering, vol. A366, pp. 164-174, 2004.

[22] R. C. Garvie and P. S. Nicholson, Journal of the American Ceramic Society, vol. 55, p. 303, 1972.

[23] B. D. Cullity, Elements of X-Ray Diffraction: Addison-Wesley, 1978.

[24] G. K. Williamson and W. H. Hall, "X-Ray Line Broadening from Filed Aluminum and Wolfram*," Acta Metallurgica, vol. 1, pp. 22-31, 1953.

[25] V. Busser, J. Desquines, S. Fouquet, M.-C. Baietto, and J.-P. Mardon, "Modelling of corrosion induced stresses during Zircaloy-4 oxidation in air," Materials Science Forum, vol. 595-598, pp. 419-427, 2008.

[26] L. Gosmain, C. Valot, D. Ciosmak, and O. Sicardy, "Study of stress effects in the oxidation of Zircaloy-4," Solid State lonics, pp. 633-640, 2001. 
[27] R. C. Garvie, "The Occurence of Metastable Tetragonal Zirconia as a Crystalline Size Effect," The Journal of Physical Chemistry, vol. 69, pp. 1238-1243, 1965.

[28] R. C. Garvie, "Stabilization of the Tetragonal Structure in Zirconia Microcrystals," The Journal of Physical Chemistry, vol. 82, pp. 218-224, 1978.

[29] $H$. Arashi and M. Ishigame, "Raman Spectroscopic Studies of the Polymorhism in $\mathrm{ZrO}_{2}$ at High Pressures," Physical Status Solidi A, vol. 71, pp. 313-321, 1982. 